\title{
Deconvolution Method used in Improving the Depth Resolution of Three- Dimensional Images Taken by Scanning Confocal Electron Microscopy
}

\author{
X. Zhang,* M. Takeguchi, ${ }^{*} * *, * * * * *$ A. Hashimoto, ${ }^{* * *}$ K. Mitsuishi,******* and M. Shimojo $* * * *$ \\ * Advanced Science Research Laboratory, Saitama Institute of Technology, 1690 Fusaiji, Fukaya, \\ Saitama 369-0293, Japan \\ ** High Voltage Electron Microscopy Station, National Institute for Materials Science, 3-13 Sakura, \\ Tsukuba 305-0003, Japan \\ *** International Center for Young Scientists, National Institute for Materials Science, 1-2-1 \\ Sengen, Tsukuba 305-0047, Japan \\ **** Quantum Dot Research Center, National Institute for Materials Science, 3-13 Sakura, Tsukuba \\ 305-0003, Japan \\ ***** Advanced Nano Characterization Center, National Institute for Materials Science, 3-13 \\ Sakura, Tsukuba 305-0003, Japan
}

Scanning confocal electron microscopy (SCEM) is a new imaging mode for electron microscopy, which provides three-dimensional (3D) information of the sample. Frigo et al. first demonstrated SCEM imaging of an inner structure of thick samples [1]. They employed a scan-descan system that was not capable of 3D scanning. Takeguchi et al. have developed a stage scanning system to carry out 3D-scan with high resolution under a fixed electron optics configuration [2]. Using this system, not only lateral (XY) sliced images but also vertical (XZ) sliced images are obtained directly without computer tomography process that requires acquisition of a tilt-series of images. Recently, Hashimoto et al. demonstrated that the use of an annular dark field (ADF) aperture improved the depth resolution of SCEM images. They showed a reconstructed 3D image of a nano-coil with the depth resolution of about $100 \mathrm{~nm}$, which was close to the vertical probe size determined by a spherical aberration $(\mathrm{Cs}=1.0 \mathrm{~mm})$ and convergence angle (about 10mrad) [3].

Figure 1 shows the ray diagram and components of ADF-SCEM with the stage scan system, while Figs.2 (a) and (b) show lateral and vertical sliced ADF-SCEM images from a carbon film, on which $5 \mathrm{~nm} \mathrm{Au}$ particles were distributed. Images of both the carbon film and Au particles are elongated along z-direction in the vertical sliced image. In this study, we applied an image processing technique to improve the depth resolution of ADF-SCEM images. Vertically sliced ADF-SCEM images were deconvoluted with a modeled point spread function. Wiener filter was adopted to minimize the effect of noise included in the image. Resultantly, the elongation along $\mathrm{z}$ direction was reduced by $35-40 \%$, as shown in Fig.2 (c).

References

[1] S.P. Frigo, Z.H. Levine, N.J. Zaluzec, Appl. Phys. Lett. 81 (2002) 2112.

[2] M.Takeguchi et al., J. Electron Microsc. 57 (2008) 123.

[3] A. Hashimoto et al., J. Appl. Phys. 106 (2009) 086101. 


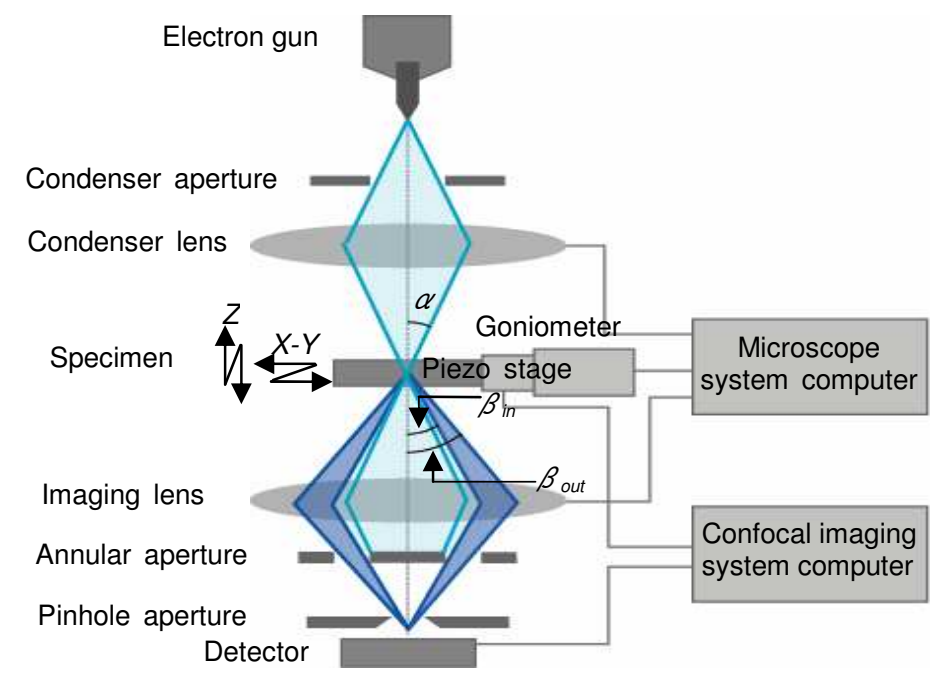

FIG. 1. Schematic diagram of ADF-SCEM equipped with a stage-scanning system.

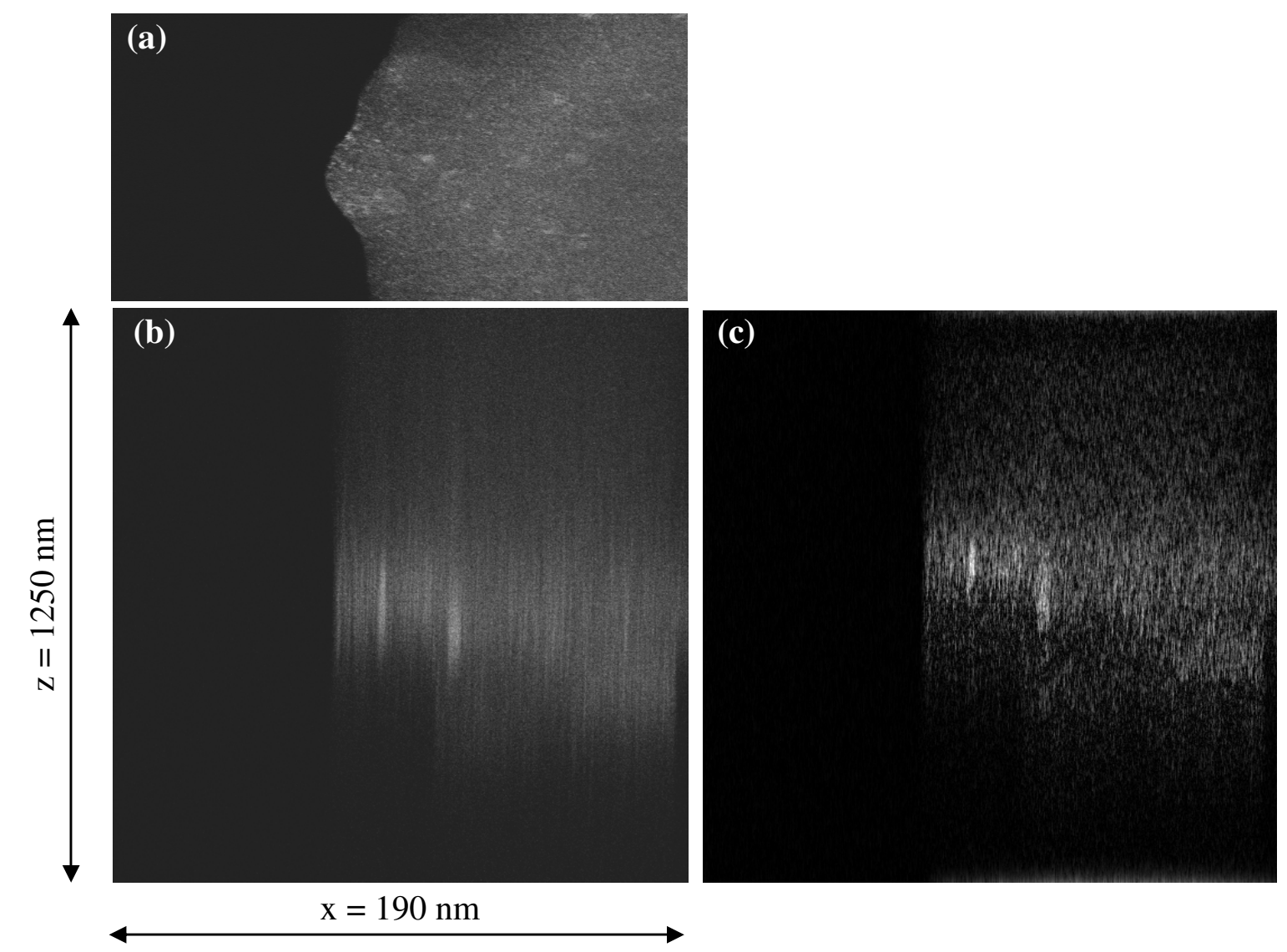

FIG. 2. X-Y scan (a) and X-Z scan (b) of ADF-SCEM from 5nm Au particles; (c) is the deconvolution result of (b). 